\title{
A New Table of Constant Weight Codes of Length Greater than 28
}

\author{
D. H. Smith, L. A. Hughes and S. Perkins \\ Division of Mathematics and Statistics \\ University of Glamorgan, Pontypridd, CF37 1DL, Wales, UK \\ \{dhsmith, lahughe1, sperkins\}@glam.ac.uk
}

Submitted: Feb 3, 2006; Accepted: May 1, 2006; Published: May 12, 2006

Mathematics Subject Classification: $94 \mathrm{~B} 60$

\begin{abstract}
Existing tables of constant weight codes are mainly confined to codes of length $n \leq 28$. This paper presents tables of codes of lengths $29 \leq n \leq 63$. The motivation for creating these tables was their application to the generation of good sets of frequency hopping lists in radio networks. The complete generation of all relevant cases by a small number of algorithms is augmented in individual cases by miscellaneous constructions. These sometimes give a larger number of codewords than the algorithms.
\end{abstract}

\section{Introduction}

$A(n, d, w)$ is the maximum possible number of binary vectors of length $n$, weight $w$ and pairwise Hamming distance no less than $d[10]$. Such a set of vectors is known as a constant weight code and the vectors are referred to as codewords. Tables of constant weight codes are given in [6] for $n \leq 28$. These tables are extended to $n \leq 65$ and sometimes above in [13], but the results are very sparse for larger values of $n$. Improved results for upper bounds are given in [2] and corresponding tables for $n \leq 28$ can be found at [14].

In this paper tables of constant weight codes are given for $29 \leq n \leq 63$. The motivation for this work was the generation of frequency hopping lists for use in assignment problems in radio networks. Large distance between codewords gives smaller overlap between lists. This leads to fewer clashes on the same frequency and so less interference. Similarly, a larger number of codewords allows larger list re-use distances in the network and again leads to lower interference. More information on the work can be found in [12] and an evaluation of assignments of the lists generated can be found in [11]. The tables given here are significantly more complete than those given in [12] and include many improvements.

The restriction $n \leq 63$ was selected as 63 is the maximum number of frequencies possible in GSM mobile telephone systems when frequency hopping is used. The range 
$3 \leq w \leq 8$ was selected for the work described in [12] as lists of length 2 are known to give no advantages when hopping and the maximum gains from frequency diversity (mitigating frequency selective fading) and interference diversity (averaging interference to ensure that the error-control coding is effective) are achieved when $w=8$. Disjoint lists lead to unsatisfactorily small list re-use distances, so the cases $d=2 w-2$ (overlap 1 ), $d=2 w-4$ (overlap 2) and $d=2 w-6$ (overlap 3) were considered. However, the tables in [13] are complete for $w=3$ and $w=4$ when $n \leq 63$, so the tables for these values (which were included in [12]) will not be presented here.

Within these ranges it was required that a constant weight code could be generated with a large number of codewords (without necessarily achieving $A(n, d, w)$ ) in all cases, using either (i) one of only a small number of algorithms suitable for Engineering application (ii) individual mathematical constructions which give further increases in the number of codewords in specific cases. Thus the tables allow a general comparison of the merits of the chosen algorithms. They also demonstrate further improvements by detailed constructions in some individual cases.

Let $\left(q_{1}, q_{2}, n_{1}, n_{2}, d\right)$ denote a mixed error-correcting code, of length $n=n_{1}+n_{2}$, where the first $n_{1}$ entries of any codeword take values from 0 to $q_{1}-1$, the next $n_{2}$ entries take values from 0 to $q_{2}-1$, and the minimal Hamming distance between different codewords is no less than $d$. In one of the construction methods in Section 2.2 the following simple result is used:

Proposition 1 Suppose that $C$ is a $\left(q_{1}, q_{2}, n_{1}, n_{2}, d\right)$ code. Then there exists a constant weight binary code $\widetilde{C}$ with $|C|$ words of length $\widetilde{n}=q_{1} n_{1}+q_{2} n_{2}$, minimum distance $2 d$, and weight $w=n_{1}+n_{2}$. This code is constructed in the following way: take each word $X=\left[x_{1} x_{2} \ldots x_{n}\right]$ of the initial $\left(q_{1}, q_{2}, n_{1}, n_{2}, d\right)$ code and substitute each $x_{i}$ in it by a row of $q_{1}$ entries if $i \leq n_{1}$ and by a row of $q_{2}$ entries if $i>n_{1}$. Of those entries (numbering the entries starting from 1 ) the entry with number $x_{i}+1$ is equal to 1 and all other entries are 0 .

\section{Constructions}

In this section the constructions used to create the tables are described:

\subsection{Construction from permutation groups}

This construction of codes from a permutation group $G$ generated by a single permutation is taken from [6]. Initially all orbits of $G$ are determined, starting from orbits of codewords of weight 1 . Consider a complete set of binary vectors of length $n$ and weight $i$, each of which is a lexicographically maximal representative of its orbit. Suppose also that these are arranged in decreasing lexicographic order. From each vector, new vectors of weight $i+1$ are generated by converting a single 0 to a 1 in all possible ways. For each vector generated, determine whether it is lexicographically maximal over its orbit. If it is, record the vector, otherwise discard it. 
For $t=w-d / 2+1$, a matrix $B$ with columns indexed by orbits of weight $w$ and with rows indexed by orbits of weight $t$ can be formed. $B$ specifies how often a representative vector of weight $t$ is covered by the vectors in a given orbit of weight $w$. Orbits of weight $w$ for which the corresponding row of $B$ contains an entry greater than 1 can be discarded (as two elements of the constant weight code will have overlap greater than $w-d / 2$ ).

The remaining orbits of weight $w$ are represented by the vertices of a weighted graph, with the vertex labelled by the number of codewords in the set. Two vertices are joined if the pair does not conflict with the minimum distance condition. A maximum clique algorithm is then used to find the maximum weighted clique in this graph which, from the orbits represented by its vertices, gives the maximum constant weight code for this $G$.

Cyclic cases (with the permutation a cycle of length $n$ ), extended cyclic cases (with the permutation a cycle of length $n-1$ ) and (for $n=2 s$ ) quasi-cyclic cases (with a permutation $(12 \ldots s)(s+1 \ldots 2 s))$ were considered. For $29 \leq n \leq 63$ these provided a challenging set of computations. The maximum clique software used was based on the algorithm in [7]. The computation could fail for reasons of either memory or run time. In some cases $\left(\right.$ marked ${ }^{*}$ ) only an incomplete clique search was possible. Sometimes these incomplete searches gave new best results. A poor result from an incomplete search is a reflection of the infeasibility of the algorithm rather than its ineffectiveness. If the clique search will not terminate it is often useful to apply the maximum clique algorithm with several different orderings by vertex degrees. This sometimes leads to a larger clique being found quickly. Sometimes a clique of size 1 or 2 gives a good result and the maximum clique algorithm is unnecessary.

Cyclic cases are denoted $C C$ in the tables; extended cyclic cases are denoted $E C$ in the tables and quasi-cyclic cases are denoted $Q C$ in the tables.

\subsection{Lexicographic search for mixed or non-binary codes}

In this method parameters for a suitable non-binary or mixed code (so that $n=n_{1} q_{1}$ or $n=n_{1} q_{1}+n_{2} q_{2}$ ) are determined and a lexicographic search [9] for such a code is performed. The code found by the search which has the maximum number of codewords is then used in Proposition 1. Results are given in the columns marked NB-Mix. Constant weight codes constructed by this method cannot be expected to be particularly good; in just four cases this method gave the best result. However, the method is easily the fastest of those used (finding an example for every case $9 \leq n \leq 63$ in a single run of under 24 hours on a $400 \mathrm{MHz}$ Pentium PC with $128 \mathrm{Mb}$ of memory). In the frequency hopping application it may prove useful that the 1's in the codewords constructed appear once in every $q_{1}$ or $q_{2}$ consecutive positions.

\subsection{Binary lexicographic search}

Several variations of binary lexicographic search [9] are possible. Here binary vectors of length $n$ and weight $w$ are arranged in either forward or reverse lexicographic order. A single vector is used as a seed vector and the other vectors are selected in turn and added 
to the code if they satisfy the necessary distance condition. This search is usually faster than the permutation group construction, but may take over a day of computation in the largest cases. However, it always proved possible to complete both the forward and the reverse search. The better of the two results is given in the tables in the column marked $B-L e x$, and annotated $(R)$ if it is obtained by reverse search.

\subsection{Random search}

If the run time for lexicographic search is unsatisfactory, codes can be constructed randomly. Words of weight $w$ are chosen randomly and tested to see whether they meet the required distance condition with previously chosen codewords. If they do, they are added to the code. The ultimate number of codewords selected is smaller but more codewords can be obtained in a limited search. No results are presented as they are almost always much worse than binary lexicographic search.

\subsection{Miscellaneous constructions}

Miscellaneous constructions of four types were considered and lead to codes as follows:

- codes with $29 \leq n \leq 63$ constructed by application of some method described in [6],

- other codes taken from [13] where a construction is given,

- other codes taken from the literature,

- codes constructed by the authors.

In all cases the method is indicated in the Key. All methods in [6] were considered when attempting to construct a new best code. Of course it was impossible to be comprehensive in selecting a permutation group or code to be the basis of some of these constructions.

\section{A table of constant weight codes}

The results are given in Tables 1- 12. The tables also display the Johnson upper bound [6] (in the column marked $U B$ in the tables). The reader is referred to [2] for possible improvements to this bound. The best result is indicated in bold in the tables. The column NewBest indicates a new best result. The meaning of the annotations is given in the Key.

The tables show the relative merits of the general algorithms in terms of the number of codewords generated, with the permutation group construction being generally the best of the algorithms used, followed by binary lexicographic search. Non-binary or mixed lexicographic search is certainly the fastest method, but binary lexicographic search will generally be preferred if the permutation group construction is too slow. 


\section{KEY}

- $\mathrm{A}_{n}$ - From a code above (or from [6]) of length $n$.

- $\mathrm{B}$ - Using $A(65,8,8)=65520$ [15], equation $5($ ii) of [6] gives $A(64,8,8)=57456$.

Comment. $A(64,8,8)=57456, A(65,8,8)=65520$. The code realising $A(65,8,8)=65520$ consists of two orbits of length 32760 under $P S L_{2}(64)$. Taken from [13].

- BE - via Baker's elliptic semi-plane, a \{7\}-GDD of type $3^{15}$, [4], p.191 [8], [13].

Comment. $A(45,12,7)=45$. Given a partition of the 45 points into 15 groups of size 3 then in the GDD two of the 45 points are either in a (single) block (of 7 points) or in a single group, but not both. Thus the overlap is at most 1. Counting we have $\left(\begin{array}{c}45 \\ 2\end{array}\right)=b .\left(\begin{array}{l}7 \\ 2\end{array}\right)+13 .\left(\begin{array}{l}3 \\ 2\end{array}\right)$ so $b=45$. Taken from [13].

- C - Theorem 1 of [3].

Comment. $A(33,8,5)=44, A(34,8,5)=47$.

- D - Construction I from [1].

Comment. $A(55,8,5)=121, A(42,8,6)=343$. In the first case $p=11, n=5$, $k=2$ and in the second case $p=7, n=6, k=3$.

- EH - Words of weight 5 in a translate of the $(32,26)$ Extended Hamming Code, using a vector of weight 1 .

Comment. $A(32,4,5)=6293$.

- Eq. $x$-Equation $x$ of [6].

- $\mathrm{H}_{n}$ - Adding words to a code above (or from [6]) of length $n$.

- M - Manual construction.

- NB - Construct the code realising $A_{5}(8,7)=10[5]$ and apply Proposition 1.

Comment. $A(40,14,8)=44$.

- P - Words of weight 6 in the Preparata code of length 64 .

- R - Reverse binary lexicographic search.

- Th. $y$ - Theorem $y$ of $[6]$.

- S - Completing a $(28,4,1)$ RBIBD (p. 90, [8]) gives a partially balanced design with 63 blocks of size 5 and one of size 9 . This last block can be replaced by two blocks of size 5, p90 [8], [13].

Comment. $A(37,8,5)=65$. A parallel class of a $(28,4,1)$ design consists of 7 blocks forming a partition of the 28 points. The 63 blocks form 9 parallel classes. Add a point 29 to each block of the first parallel class, 30 to each block of the second,... 37 to each block of the ninth. Finally, add two extra blocks 29,30,31,32,33 and $33,34,35,36,37$. Taken from [13].

- SS - A Steiner system.

- * - Clique search was incomplete. 


\begin{tabular}{|c|c|c|c|c|c|c|}
\hline$n$ & $C C, E C, Q C$ & $N B-M i x$ & $B-$ Lex & Misc & $U B$ & NewBest \\
\hline 29 & $3770, * 3591,-$ & 816 & $3731(\mathrm{R})$ & 4095 (Eq. 31) & 4750 & \\
\hline 30 & & 976 & $4459(\mathrm{R})$ & 4751 (Eq. 31) & 5262 & \\
\hline 31 & & 1136 & $5313(\mathrm{R})$ & 5481 (Eq. 31) & 6274 & \\
\hline 32 & & 1324 & $6293(\mathrm{R})$ & $6293(\mathrm{EH})$ & 6944 & \\
\hline 33 & & 1544 & 6503 & 7192 (Eq. 31) & 8184 & \\
\hline 34 & & 1801 & 7051 & 8184 (Eq. 31) & 8976 & \\
\hline 35 & & 2101 & 7159 & 9276 (Eq. 31) & 10472 & \\
\hline 36 & & 2401 & 7881 & 10472 (Eq. 31) & 11397 & \\
\hline 37 & & 2744 & $8353(\mathrm{R})$ & 11781 (Eq. 31) & 13186 & \\
\hline 38 & & 3136 & 9259 & 13209 (Eq. 31) & 14341 & \\
\hline 39 & & 3584 & 10168 & 14763 (Eq. 31) & 16450 & \\
\hline 40 & & 4096 & 11334 & 16451 (Eq. 31) & 17784 & \\
\hline 41 & & 4096 & 12598 & 18278 (Eq. 31) & 20254 & \\
\hline 42 & & 4160 & 14156 & 20254 (Eq. 31) & 21781 & \\
\hline 43 & & 4288 & 15831 & 22386 (Eq. 5(ii)) & 24647 & \\
\hline 44 & & 4481 & 17635 & 25256 (Eq. 5(ii)) & 26488 & \\
\hline 45 & & 4741 & 19657 & 28413 (Eq. 5(ii)) & 29799 & \\
\hline 46 & & 5001 & 21940 & 31878 (Eq. 5(ii)) & 31878 & \\
\hline 47 & & 5328 & 24488 & 35673 (SS) & 35673 & \\
\hline 48 & & 5724 & $27273(\mathrm{R})$ & 35674 (Th. 18) & 38006 & \\
\hline 49 & & 6192 & $30348(\mathrm{R})$ & 38916 (Eq. 31) & 42336 & \\
\hline 50 & & 6736 & 33667 & 42376 (Eq. 31) & 45080 & \\
\hline 51 & & 7280 & $37092(\mathrm{R})$ & 46060 (Eq. 31) & 49980 & \\
\hline 52 & & 7900 & $40928(\mathrm{R})$ & 49980 (Eq. 31) & 53040 & \\
\hline 53 & & 8600 & $45101(\mathrm{R})$ & 54145 (Eq. 31) & 58565 & \\
\hline 54 & & 9385 & $49633(\mathrm{R})$ & 58565 (Eq. 31) & 61959 & \\
\hline 55 & & 10000 & $54547(\mathrm{R})$ & 63251 (Eq. 31) & 68156 & \\
\hline 56 & & 10000 & $59867(\mathrm{R})$ & 68211 (Eq. 31) & 72072 & \\
\hline 57 & & 10000 & $65618(\mathrm{R})$ & 73458 (Eq. 31) & 79002 & \\
\hline 58 & & 10000 & $71722(\mathrm{R})$ & 79002 (Eq. 31) & 83311 & \\
\hline 59 & & 10000 & $78302(\mathrm{R})$ & 84854 (Eq. 31) & 91025 & \\
\hline 60 & & 10000 & $85386(\mathrm{R})$ & 91026 (Eq. 31) & 95748 & \\
\hline 61 & & 10000 & $93003(\mathrm{R})$ & 97527 (Eq. 31) & 104310 & \\
\hline 62 & & 10000 & $101123(\mathrm{R})$ & 104371 (Eq. 31) & 109678 & \\
\hline 63 & & 10000 & $109833(\mathrm{R})$ & 111569 (Eq. 31) & 119133 & \\
\hline
\end{tabular}

Table 1: Comparison of Results $d=4, w=5$. 


\begin{tabular}{|c|c|c|c|c|c|c|}
\hline$n$ & $C C, E C, Q C$ & $N B-M i x$ & $B-L e x$ & Misc & $U B$ & NewBest \\
\hline 29 & $\overline{2090, * 287,-}$ & $\overline{c 100}$ & 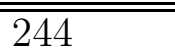 & & 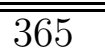 & $\overline{\overline{\mathrm{Y}}}$ \\
\hline 30 & $306,{ }^{*} \mathbf{3 1 9},{ }^{*} 315$ & 112 & 271 (R) & & 390 & $\mathrm{Y}$ \\
\hline 31 & $341,{ }^{*} \mathbf{3 6 6},-$ & 127 & $311(\mathrm{R})$ & & 415 & $\mathrm{Y}$ \\
\hline 32 & $384, * 403, * 384$ & 137 & 340 & & 492 & $\mathrm{Y}$ \\
\hline 33 & $429, * 424,-$ & 150 & $379(\mathrm{R})$ & & 528 & $\mathrm{Y}$ \\
\hline 34 & $476, * \mathbf{4 9 5}, * 459$ & 162 & 413 & & 557 & $\mathrm{Y}$ \\
\hline 35 & $532,{ }^{*} 510,-$ & 182 & $456(\mathrm{R})$ & & 651 & $\mathrm{Y}$ \\
\hline 36 & $576,{ }^{*} 567,{ }^{*} 504$ & 202 & 496 & & 691 & Y \\
\hline 37 & $629,{ }^{*} 621,-$ & 219 & 542 & & 732 & $\mathrm{Y}$ \\
\hline 38 & $684,{ }^{*} 666,-$ & 200 & $591(\mathrm{R})$ & & 843 & $\mathrm{Y}$ \\
\hline 39 & $741, * 722,-$ & 224 & 638 & & 889 & Y \\
\hline 40 & & 256 & $694(\mathrm{R})$ & $741\left(\mathrm{~A}_{39}\right)$ & 936 & $\mathrm{Y}$ \\
\hline 41 & & 288 & $755(\mathrm{R})$ & & 1066 & $\mathrm{Y}$ \\
\hline 42 & & 288 & 817 & & 1117 & $\mathrm{Y}$ \\
\hline 43 & & 321 & 874 & & 1169 & $\mathrm{Y}$ \\
\hline 44 & & 346 & $941(\mathrm{R})$ & & 1320 & $\mathrm{Y}$ \\
\hline 45 & & 372 & 1009 & & 1386 & $\mathrm{Y}$ \\
\hline 46 & & 405 & $1097(\mathrm{R})$ & & 1444 & $\mathrm{Y}$ \\
\hline 47 & & 430 & 1172 & & 1616 & $\mathrm{Y}$ \\
\hline 48 & & 464 & $1254(\mathrm{R})$ & & 1689 & Y \\
\hline 49 & & 504 & $1343(\mathrm{R})$ & & 1764 & $\mathrm{Y}$ \\
\hline 50 & & 532 & 1429 & & 1960 & $\mathrm{Y}$ \\
\hline 51 & & 619 & $1517(\mathrm{R})$ & & 2040 & Y \\
\hline 52 & & 668 & 1617 & & 2121 & $\mathrm{Y}$ \\
\hline 53 & & 731 & 1719 & & 2342 & $\mathrm{Y}$ \\
\hline 54 & & 776 & 1822 & & 2430 & $\mathrm{Y}$ \\
\hline 55 & & 824 & $1924(\mathrm{R})$ & 1936 (Eq. 5(ii)) & 2519 & \\
\hline 56 & & 736 & 2036 & 2125 (Eq. 5(ii)) & 2766 & \\
\hline 57 & & 674 & 2162 & 2329 (Eq. 5(ii)) & 2872 & \\
\hline 58 & & 576 & $2280(\mathrm{R})$ & 2548 (Eq. 5(ii)) & 2969 & \\
\hline 59 & & 576 & 2397 & 2783 (Eq. 5(ii)) & 3245 & \\
\hline 60 & & 576 & 2531 & 3036 (Eq. 5(ii)) & 3360 & \\
\hline 61 & & 898 & 2665 & 3306 (Eq. 5(ii)) & 3477 & \\
\hline 62 & & 1017 & $2801(\mathrm{R})$ & 3596 (Eq. 5(ii)) & 3782 & \\
\hline 63 & & 1122 & $2952(\mathrm{R})$ & 3906 (Eq. 5(i)P) & 3906 & \\
\hline
\end{tabular}

Table 2: Comparison of Results $d=6, w=5$. 


\begin{tabular}{|c|c|c|c|c|c|c|}
\hline$n$ & $C C, E C, Q C$ & $N B-M i x$ & $B-$ Lex & Misc & $U B$ & NewBest \\
\hline$\overline{\overline{29}}$ & $8999,{ }^{*} 882,-$ & $\overline{2026}$ & $853(\mathrm{R})$ & 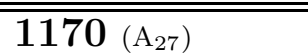 & $\bar{~} 1459$ & \\
\hline 30 & & 265 & $1005(\mathrm{R})$ & $1179\left(\mathrm{H}_{27}\right)$ & 1825 & Y \\
\hline 31 & & 303 & $1163(\mathrm{R})$ & $1205\left(\mathrm{H}_{27}\right)$ & 2015 & Y \\
\hline 32 & & 353 & $1331(\mathrm{R})$ & & 2213 & \\
\hline 33 & & 412 & $1528(\mathrm{R})$ & & 2706 & Y \\
\hline 34 & & 468 & 1740 & & 2992 & Y \\
\hline 35 & & 538 & $1973(\mathrm{R})$ & & 3249 & Y \\
\hline 36 & & 618 & 2240 & & 3906 & Y \\
\hline 37 & & 676 & $2539(\mathrm{R})$ & & 4261 & Y \\
\hline 38 & & 762 & $2836(\mathrm{R})$ & & 4636 & Y \\
\hline 39 & & 842 & 3167 & & 5479 & Y \\
\hline 40 & & 944 & 3545 & & 5926 & Y \\
\hline 41 & & 1049 & 3964 & & 6396 & Y \\
\hline 42 & & 1175 & 4397 & & 7462 & Y \\
\hline 43 & & 1284 & 4860 & & 8005 & Y \\
\hline 44 & & 1402 & 5378 & & 8572 & Y \\
\hline 45 & & 1368 & $5933(\mathrm{R})$ & & 9900 & Y \\
\hline 46 & & 1568 & 6521 & & 10626 & Y \\
\hline 47 & & 1792 & $7160(\mathrm{R})$ & & 11311 & Y \\
\hline 48 & & 2048 & 7845 & & 12928 & Y \\
\hline 49 & & 2190 & 8568 & & 13793 & Y \\
\hline 50 & & 2366 & $9348(\mathrm{R})$ & & 14700 & $\mathrm{Y}$ \\
\hline 51 & & 2577 & 10175 & & 16660 & Y \\
\hline 52 & & 2807 & 11064 & 11316 (Eq. 5(ii)) & 17680 & \\
\hline 53 & & 3055 & 12025 & 12760 (Eq. 5(ii)) & 18735 & \\
\hline 54 & & 3346 & $13017(\mathrm{R})$ & 14355 (Eq. 5(ii)) & 21078 & \\
\hline 55 & & 3605 & 14091 & 16112 (Eq. 5(ii)) & 22275 & \\
\hline 56 & & 3881 & $15221(\mathrm{R})$ & 18045 (Eq. 5(ii)) & 23510 & \\
\hline 57 & & 4210 & 16422 & 20167 (Eq. 5(ii)) & 26277 & \\
\hline 58 & & 4532 & 17683 & 22493 (Eq. 5(ii)) & 27762 & \\
\hline 59 & & 4854 & $19028(\mathrm{R})$ & 25039 (Eq. 5(ii)) & 29195 & \\
\hline 60 & & 5258 & 20431 & 27821 (Eq. 5(ii)) & 32450 & \\
\hline 61 & & 5638 & 21940 & 30856 (Eq. 5(ii)) & 34160 & \\
\hline 62 & & 6026 & 23493 & 34162 (Eq. 5(ii)) & 35929 & \\
\hline 63 & & 6473 & $25185(\mathrm{R})$ & 37758 (Eq. 5(ii)P) & 39711 & \\
\hline
\end{tabular}

Table 3: Comparison of Results $d=6, w=6$. 


\begin{tabular}{|c|c|c|c|c|c|c|}
\hline$n$ & $C C, E C, Q C$ & $N B-M i x$ & $B-L e x$ & Misc & $U B$ & NewBest \\
\hline 29 & 29, 35,- & 18 & 27 & & 40 & $\overline{\bar{Y}}$ \\
\hline 30 & $\mathbf{3 6}, 29, \mathbf{3 6}$ & 18 & 29 & & 42 & \\
\hline 31 & $31, \mathbf{3 6},-$ & 20 & 32 & & 43 & \\
\hline 32 & $32,31,32$ & 23 & 34 & 38 (Eq. 5(ii)) & 44 & \\
\hline 33 & $33,40,-$ & 24 & 36 & $44(\mathrm{C})$ & 52 & \\
\hline 34 & $34,33,34$ & 25 & 38 & $47(\mathrm{C})$ & 54 & \\
\hline 35 & $42,34,-$ & 27 & 41 & 50 (Eq. 5(ii)) & 56 & \\
\hline 36 & $36,42,54$ & 31 & 44 & 57 (Eq. 5(ii)) & 57 & \\
\hline 37 & $37,45,-$ & 31 & 47 & $65(\mathrm{~S})$ & 66 & \\
\hline 38 & $38,37,57$ & 32 & 50 & $65\left(\mathrm{~A}_{37}\right)$ & 68 & \\
\hline 39 & $39,38,-$ & 32 & 52 & $65\left(\mathrm{~A}_{37}\right)$ & 70 & \\
\hline 40 & $48,39,64$ & 32 & 57 & 72 (Eq. 5(ii)) & 72 & \\
\hline 41 & $82,58,-$ & 32 & 60 & 82 (SS) & 82 & \\
\hline 42 & $42,82,{ }^{*} 63$ & 33 & 62 & & 84 & \\
\hline 43 & $\mathbf{8 6}, 42,-$ & 35 & 67 & & 86 & \\
\hline 44 & $88,86,{ }^{*} 88$ & 33 & 68 & & 88 & \\
\hline 45 & $54,55,-$ & 33 & 73 & 99 (SS) & 99 & \\
\hline 46 & $92,54,{ }^{*} 92$ & 36 & 76 & $99\left(\mathrm{~A}_{45}\right)$ & 101 & \\
\hline 47 & $94,92,-$ & 40 & 80 & $99\left(\mathrm{~A}_{45}\right)$ & 103 & \\
\hline 48 & $96,94, * 96$ & 40 & 81 & $99\left(\mathrm{~A}_{45}\right)$ & 105 & \\
\hline 49 & $98, \mathbf{1 0 8},-$ & 46 & 85 & & 117 & Y \\
\hline 50 & $\mathbf{1 1 0}, 98, * 80$ & 46 & 89 & & 120 & \\
\hline 51 & $102, \mathbf{1 1 0},-$ & 52 & 91 & & 122 & \\
\hline 52 & $104,102, * 54$ & 54 & 96 & $110\left(\mathrm{~A}_{51}\right)$ & 124 & \\
\hline 53 & $106, \mathbf{1 1 7},-$ & 57 & 100 & & 137 & \\
\hline 54 & $108, * 106, * 54$ & 65 & 105 & $117\left(\mathrm{~A}_{53}\right)$ & 140 & \\
\hline 55 & $110, * 108,-$ & 68 & 107 & 121 (D) & 143 & \\
\hline 56 & $112,{ }^{*} \mathbf{1 2 1},-$ & 65 & 112 & & 145 & \\
\hline 57 & $114, * 70,-$ & 60 & 117 & 129 (Eq. 5(ii)) & 159 & \\
\hline 58 & $116, * 114,-$ & 56 & 119 & 141 (Eq. 5(ii)) & 162 & \\
\hline 59 & $118, * 116,-$ & 48 & 123 & 154 (Eq. 5(ii)) & 165 & \\
\hline 60 & $120, * 118,-$ & 48 & 122 & 168 (Eq. 5(ii)) & 168 & \\
\hline 61 & $61, * 75,-$ & 56 & 131 & 183 (SS) & 183 & \\
\hline 62 & $* 124, * 122,-$ & 63 & 136 & $183\left(\mathrm{~A}_{61}\right)$ & 186 & \\
\hline 63 & $* 126,{ }^{*} 124,-$ & 71 & 137 & $183\left(\mathrm{~A}_{61}\right)$ & 189 & \\
\hline
\end{tabular}

Table 4: Comparison of Results $d=8, w=5$. 


\begin{tabular}{|c|c|c|c|c|c|c|}
\hline$n$ & $C C, E C, Q C$ & $N B-M i x$ & $B-L e x$ & Misc & $U B$ & NewBest \\
\hline 29 & 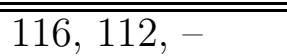 & 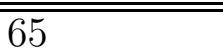 & 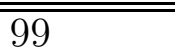 & 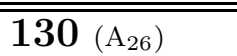 & $\overline{c 159}$ & \\
\hline 30 & $125,116, * 105$ & 67 & $115(\mathrm{R})$ & $131\left(\mathrm{H}_{26}\right)$ & 200 & Y \\
\hline 31 & $155,155,-$ & 69 & $125(\mathrm{R})$ & 156 (Eq. 5(ii)) & 217 & $\mathrm{Y}$ \\
\hline 32 & $\mathbf{1 9 2 ,} 155,{ }^{*} 104$ & 73 & $131(\mathrm{R})$ & & 229 & $\mathrm{Y}$ \\
\hline 33 & $-, 160,-$ & 76 & $139(\mathrm{R})$ & $192\left(\mathrm{~A}_{32}\right)$ & 242 & $\mathrm{Y}$ \\
\hline 34 & & 76 & $152(\mathrm{R})$ & $192\left(\mathrm{~A}_{32}\right)$ & 294 & $\mathrm{Y}$ \\
\hline 35 & & 80 & 168 & $192\left(\mathrm{~A}_{32}\right)$ & 315 & $\mathrm{Y}$ \\
\hline 36 & & 88 & $184(\mathrm{R})$ & $193\left(\mathrm{H}_{32}\right)$ & 336 & \\
\hline 37 & & 95 & 199 & & 351 & \\
\hline 38 & & 109 & 222 & & 418 & \\
\hline 39 & & 118 & $244(\mathrm{R})$ & & 442 & \\
\hline 40 & & 128 & $275(\mathrm{R})$ & & 466 & \\
\hline 41 & & 137 & $285(\mathrm{R})$ & 294 (Eq. 5(ii)) & 492 & \\
\hline 42 & & 147 & 307 & 343 (D) & 574 & \\
\hline 43 & & 160 & 332 & $343\left(\mathrm{H}_{42}\right)$ & 602 & \\
\hline 44 & & 164 & $355(\mathrm{R})$ & & 630 & \\
\hline 45 & & 178 & 381 & & 660 & $\mathrm{Y}$ \\
\hline 46 & & 200 & $411(\mathrm{R})$ & & 759 & \\
\hline 47 & & 224 & $440(\mathrm{R})$ & & 791 & Y \\
\hline 48 & & 256 & $477(\mathrm{R})$ & & 824 & $\mathrm{Y}$ \\
\hline 49 & & 256 & 501 & & 857 & $\mathrm{Y}$ \\
\hline 50 & & 264 & 542 & & 975 & $\mathrm{Y}$ \\
\hline 51 & & 253 & 576 & & 1020 & $\mathrm{Y}$ \\
\hline 52 & & 271 & $609(\mathrm{R})$ & & 1057 & $\mathrm{Y}$ \\
\hline 53 & & 289 & 650 & & 1095 & $\mathrm{Y}$ \\
\hline 54 & & 314 & 682 & & 1233 & $\mathrm{Y}$ \\
\hline 55 & & 334 & 729 & & 1283 & $\mathrm{Y}$ \\
\hline 56 & & 347 & 766 & & 1334 & $\mathrm{Y}$ \\
\hline 57 & & 376 & 830 & & 1377 & $\mathrm{Y}$ \\
\hline 58 & & 402 & 872 & & 1537 & $\mathrm{Y}$ \\
\hline 59 & & 420 & 935 & & 1593 & $\mathrm{Y}$ \\
\hline 60 & & 446 & 982 & & 1650 & $\mathrm{Y}$ \\
\hline 61 & & 471 & 1028 & & 1708 & $\mathrm{Y}$ \\
\hline 62 & & 505 & 1079 & & 1891 & $\mathrm{Y}$ \\
\hline 63 & & 531 & 1143 & & 1953 & $\mathrm{Y}$ \\
\hline
\end{tabular}

Table 5: Comparison of Results $d=8, w=6$. 


\begin{tabular}{|c|c|c|c|c|c|c|}
\hline$n$ & $C C, E C, Q C$ & $N B-M i x$ & $B-$ Lex & Misc & $U B$ & NewBest \\
\hline 29 & 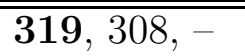 & 64 & 300 & & "617 & $\overline{\bar{Y}}$ \\
\hline 30 & & 76 & $327(\mathrm{R})$ & & 681 & \\
\hline 31 & & 86 & 363 & & 885 & Y \\
\hline 32 & & 104 & $403(\mathrm{R})$ & & 992 & \\
\hline 33 & & 122 & 444 & & 1079 & Y \\
\hline 34 & & 150 & 498 & & 1175 & $\mathrm{Y}$ \\
\hline 35 & & 165 & $555(\mathrm{R})$ & & 1470 & \\
\hline 36 & & 187 & $622(\mathrm{R})$ & & 1620 & \\
\hline 37 & & 214 & 696 & & 1776 & \\
\hline 38 & & 241 & $785(\mathrm{R})$ & & 1905 & \\
\hline 39 & & 278 & $869(\mathrm{R})$ & & 2328 & \\
\hline 40 & & 310 & 977 & & 2525 & Y \\
\hline 41 & & 350 & $1095(\mathrm{R})$ & & 2729 & \\
\hline 42 & & 390 & $1206(\mathrm{R})$ & & 2952 & \\
\hline 43 & & 425 & 1347 & & 3526 & Y \\
\hline 44 & & 474 & 1478 & & 3784 & Y \\
\hline 45 & & 522 & 1639 & & 4050 & Y \\
\hline 46 & & 578 & 1795 & & 4337 & \\
\hline 47 & & 631 & 1987 & & 5096 & Y \\
\hline 48 & & 699 & $2173(\mathrm{R})$ & & 5424 & Y \\
\hline 49 & & 766 & 2376 & & 5768 & Y \\
\hline 50 & & 835 & $2603(\mathrm{R})$ & & 6121 & Y \\
\hline 51 & & 896 & 2839 & & 7103 & Y \\
\hline 52 & & 970 & 3101 & & 7577 & Y \\
\hline 53 & & 1072 & $3376(\mathrm{R})$ & & 8003 & Y \\
\hline 54 & & 1376 & $3651(\mathrm{R})$ & & 8447 & Y \\
\hline 55 & & 1792 & 3941 (R) & & 9687 & Y \\
\hline 56 & & 2048 & 4270 & & 10264 & Y \\
\hline 57 & & 2048 & 4625 & & 10862 & $\mathrm{Y}$ \\
\hline 58 & & 1557 & $4971(\mathrm{R})$ & & 11409 & Y \\
\hline 59 & & 1675 & $5384(\mathrm{R})$ & & 12954 & Y \\
\hline 60 & & 1780 & 5770 & & 13654 & Y \\
\hline 61 & & 1910 & $6223(\mathrm{R})$ & & 14378 & Y \\
\hline 62 & & 2078 & 6693 & & 15128 & $\mathrm{Y}$ \\
\hline 63 & & 2227 & 7171 & 7182 (Eq. 5(i)B) & 17019 & \\
\hline
\end{tabular}

Table 6: Comparison of Results $d=8, w=7$. 


\begin{tabular}{|c|c|c|c|c|c|c|}
\hline$n$ & $C C, E C, Q C$ & $N B-M i x$ & $B-L e x$ & Misc & $U B$ & NewBest \\
\hline 29 & $0,0,-$ & 12 & 15 & 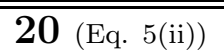 & 24 & \\
\hline 30 & $5,0,15$ & 13 & $17(\mathrm{R})$ & 25 (Eq. 5(ii)) & 25 & \\
\hline 31 & $31,11,-$ & 14 & 19 & 31 (SS) & 31 & \\
\hline 32 & $0,31,16$ & 15 & 23 & $31\left(\mathrm{~A}_{31}\right)$ & 32 & \\
\hline 33 & $0,0,-$ & 17 & 23 & $31\left(\mathrm{~A}_{31}\right)$ & 33 & \\
\hline 34 & $0,0,17$ & 19 & 26 & $31\left(\mathrm{~A}_{31}\right)$ & 34 & \\
\hline 35 & $35,0,-$ & 22 & 26 & & 35 & \\
\hline 36 & $\mathbf{3 6}, 35, \mathbf{3 6}$ & 22 & 27 & & 42 & Y \\
\hline 37 & $37,36,-$ & 22 & 30 & & 43 & Y \\
\hline 38 & $38,37,38$ & 25 & 31 & & 44 & Y \\
\hline 39 & $\mathbf{3 9}, 38,-$ & 26 & 32 & & 45 & $\mathrm{Y}$ \\
\hline 40 & $\mathbf{4 0}, 39, \mathbf{4 0}$ & 28 & 35 & & 46 & $\mathrm{Y}$ \\
\hline 41 & $41,40,-$ & 31 & 34 & 42 (Eq. 5(ii)) & 54 & Y \\
\hline 42 & $49,41,49$ & 32 & 38 & & 56 & $\mathrm{Y}$ \\
\hline 43 & $43, \mathbf{4 9},-$ & 34 & 39 & & 57 & Y \\
\hline 44 & $44,43, * 44$ & 29 & 43 & $49\left(\mathrm{~A}_{43}\right)$ & 58 & $\mathrm{Y}$ \\
\hline 45 & $45,44,-$ & 31 & 44 & $49\left(\mathrm{~A}_{43}\right)$ & 60 & $\mathrm{Y}$ \\
\hline 46 & $46, \mathbf{5 4}, 23$ & 33 & 46 & & 69 & $\mathrm{Y}$ \\
\hline 47 & $47,46,-$ & 32 & 48 & 56 (Th. 21) & 70 & \\
\hline 48 & $\mathbf{5 6}, 47,24$ & 32 & 47 & & 72 & \\
\hline 49 & $49, \mathbf{5 6},-$ & 36 & 50 & & 73 & \\
\hline 50 & $50,49,25$ & 32 & 50 & $56\left(\mathrm{~A}_{49}\right)$ & 75 & \\
\hline 51 & $51, \mathbf{6 0},-$ & 36 & 55 & & 85 & Y \\
\hline 52 & $52,51,26$ & 40 & 59 & $60\left(\mathrm{~A}_{51}\right)$ & 86 & Y \\
\hline 53 & $53,52,-$ & 45 & 63 & & 88 & Y \\
\hline 54 & $63,53,-$ & 48 & 65 & & 90 & $\mathrm{Y}$ \\
\hline 55 & $55,63,-$ & 49 & 68 & & 91 & Y \\
\hline 56 & $56,66,-$ & 48 & 70 & & 102 & Y \\
\hline 57 & $57,56,-$ & 52 & 69 & $70\left(\mathrm{~A}_{56}\right)$ & 104 & Y \\
\hline 58 & $58,57,-$ & 58 & 72 & & 106 & Y \\
\hline 59 & $59,58,-$ & 60 & 77 & & 108 & Y \\
\hline 60 & $70,59,-$ & 62 & 79 & & 110 & Y \\
\hline 61 & $61,72,-$ & 65 & 83 & & 122 & Y \\
\hline 62 & $62,61,-$ & 67 & 84 & & 124 & $\mathrm{Y}$ \\
\hline 63 & $\mathbf{1 2 6}, 62,-$ & 71 & 85 & & 126 & $\mathrm{Y}$ \\
\hline
\end{tabular}

Table 7: Comparison of Results $d=10, w=6$. 


\begin{tabular}{|c|c|c|c|c|c|c|}
\hline$n$ & $C C, E C, Q C$ & $N B-M i x$ & $B-$ Lex & $\overline{M i s c}$ & $U B$ & NewBest \\
\hline 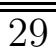 & $29,32,-$ & 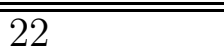 & 34 & 37 (Eq. 5(ii)) & 95 & $\overline{\overline{\mathrm{Y}}}$ \\
\hline 30 & $30,29, * 45$ & 25 & $38(\mathrm{R})$ & 48 (Eq. 5(ii)) & 102 & Y \\
\hline 31 & $62,35,-$ & 28 & 43 & & 110 & $\mathrm{Y}$ \\
\hline 32 & $32, \mathbf{6 2}, * 32$ & 30 & 47 & & 141 & Y \\
\hline 33 & $\mathbf{6 6}, 32,-$ & 34 & 54 & & 150 & Y \\
\hline 34 & $68,66, * 34$ & 36 & $60(\mathrm{R})$ & & 160 & $\mathrm{Y}$ \\
\hline 35 & $75,68,-$ & 39 & 66 & & 170 & Y \\
\hline 36 & $72, * 75, * 36$ & 44 & 75 & & 180 & Y \\
\hline 37 & $* 74, * 78,-$ & 46 & 83 & 91 (Eq. 5(ii)) & 222 & $\mathrm{Y}$ \\
\hline 38 & $-,{ }^{*} 111,-$ & 53 & $92(\mathrm{R})$ & & 233 & Y \\
\hline 39 & $-,{ }^{*} 114,-$ & 57 & $102(\mathrm{R})$ & & 245 & Y \\
\hline 40 & $-,{ }^{*} 117,-$ & 64 & $113(\mathrm{R})$ & & 257 & Y \\
\hline 41 & & 68 & 126 & & 269 & Y \\
\hline 42 & & 72 & $133(\mathrm{R})$ & & 324 & $\mathrm{Y}$ \\
\hline 43 & & 79 & 142 & 155 (Eq. 5(ii)) & 344 & \\
\hline 44 & & 83 & $155(\mathrm{R})$ & 184 (Eq. 5(ii)) & 358 & \\
\hline 45 & & 90 & $169(\mathrm{R})$ & 217 (Eq. 5(ii)) & 372 & \\
\hline 46 & & 94 & $181(\mathrm{R})$ & 255 (Eq. 5(ii)) & 394 & \\
\hline 47 & & 105 & $191(\mathrm{R})$ & 299 (Eq. 5(ii)) & 463 & \\
\hline 48 & & 112 & $209(\mathrm{R})$ & 350 (Th. 21) & 480 & \\
\hline 49 & & 118 & 224 & $350\left(\mathrm{~A}_{48}\right)$ & 504 & \\
\hline 50 & & 126 & 241 (R) & $350\left(\mathrm{~A}_{48}\right)$ & 521 & \\
\hline 51 & & 137 & 255 & $350\left(\mathrm{~A}_{48}\right)$ & 546 & \\
\hline 52 & & 146 & 272 & $350\left(\mathrm{~A}_{48}\right)$ & 631 & \\
\hline 53 & & 154 & 287 & $350\left(\mathrm{~A}_{48}\right)$ & 651 & \\
\hline 54 & & 192 & $308(\mathrm{R})$ & 351 (Th. 21) & 678 & \\
\hline 55 & & 224 & 327 (R) & $351\left(\mathrm{~A}_{54}\right)$ & 707 & \\
\hline 56 & & 256 & $348(\mathrm{R})$ & $351\left(\mathrm{~A}_{54}\right)$ & 728 & \\
\hline 57 & & 196 & $366(\mathrm{R})$ & & 830 & $\mathrm{Y}$ \\
\hline 58 & & 210 & $394(\mathrm{R})$ & & 861 & $\mathrm{Y}$ \\
\hline 59 & & 220 & $414(\mathrm{R})$ & & 893 & Y \\
\hline 60 & & 237 & 431 & & 925 & Y \\
\hline 61 & & 251 & 458 & & 958 & $\mathrm{Y}$ \\
\hline 62 & & 266 & 486 & & 1080 & $\mathrm{Y}$ \\
\hline 63 & & 277 & 514 & & 1116 & $\mathrm{Y}$ \\
\hline
\end{tabular}

Table 8: Comparison of Results $d=10, w=7$. 


\begin{tabular}{|c|c|c|c|c|c|c|}
\hline$n$ & $C C, E C, Q C$ & $N B-M i x$ & $B-$ Lex & Misc & $U B$ & NewBest \\
\hline 29 & "87, *84,-- & 28 & $7 \overline{75(\mathrm{R})}$ & & "319 & $\overline{\bar{Y}}$ \\
\hline 30 & $90, * 87, * 45$ & 36 & $89(\mathrm{R})$ & 92 (Eq. 5(ii)) & 356 & Y \\
\hline 31 & $124, * 90,-$ & 48 & 104 & & 395 & Y \\
\hline 32 & & 64 & 119 & $124\left(\mathrm{~A}_{31}\right)$ & 440 & $\mathrm{Y}$ \\
\hline 33 & & 64 & 134 & & 581 & Y \\
\hline 34 & & 69 & 156 & & 637 & Y \\
\hline 35 & & 78 & $176(\mathrm{R})$ & & 700 & Y \\
\hline 36 & & 86 & $198(\mathrm{R})$ & & 765 & $\mathrm{Y}$ \\
\hline 37 & & 98 & 223 & & 832 & $\mathrm{Y}$ \\
\hline 38 & & 109 & 249 & & 1054 & Y \\
\hline 39 & & 123 & 285 & & 1135 & Y \\
\hline 40 & & 136 & $318(\mathrm{R})$ & & 1225 & Y \\
\hline 41 & & 150 & 353 & & 1317 & Y \\
\hline 42 & & 168 & 390 & & 1412 & $\mathrm{Y}$ \\
\hline 43 & & 179 & $432(\mathrm{R})$ & & 1741 & Y \\
\hline 44 & & 198 & $484(\mathrm{R})$ & & 1892 & Y \\
\hline 45 & & 218 & 532 & & 2013 & Y \\
\hline 46 & & 242 & 590 & & 2139 & Y \\
\hline 47 & & 268 & $642(\mathrm{R})$ & & 2314 & $\mathrm{Y}$ \\
\hline 48 & & 294 & $711(\mathrm{R})$ & & 2778 & Y \\
\hline 49 & & 312 & $776(\mathrm{R})$ & & 2940 & Y \\
\hline 50 & & 338 & 852 & & 3150 & Y \\
\hline 51 & & 370 & $929(\mathrm{R})$ & & 3321 & Y \\
\hline 52 & & 391 & $1007(\mathrm{R})$ & & 3549 & Y \\
\hline 53 & & 433 & $1095(\mathrm{R})$ & & 4180 & Y \\
\hline 54 & & 469 & $1194(\mathrm{R})$ & & 4394 & Y \\
\hline 55 & & 508 & 1289 & & 4661 & $\mathrm{Y}$ \\
\hline 56 & & 544 & 1405 & & 4949 & Y \\
\hline 57 & & 579 & 1517 & & 5187 & $\mathrm{Y}$ \\
\hline 58 & & 631 & 1633 & & 6017 & Y \\
\hline 59 & & 672 & 1767 & & 6349 & Y \\
\hline 60 & & 724 & 1908 & & 6697 & $\mathrm{Y}$ \\
\hline 61 & & 792 & 2043 & & 7053 & Y \\
\hline 62 & & 992 & $2189(\mathrm{R})$ & & 7424 & Y \\
\hline 63 & & 1024 & 2352 & & 8505 & Y \\
\hline
\end{tabular}

Table 9: Comparison of Results $d=10, w=8$. 


\begin{tabular}{|c|c|c|c|c|c|c|}
\hline$n$ & $C C, E C, Q C$ & $N B-M i x$ & $B-L e x$ & Misc & $U B$ & NewBest \\
\hline 29 & $0,4,-$ & 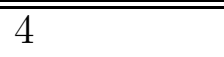 & 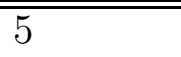 & $8(\mathrm{M})$ & 16 & $\overline{\mathrm{Y}}$ \\
\hline 30 & $0,0,0$ & 5 & 6 & $8\left(\mathrm{~A}_{29}\right)$ & 17 & Y \\
\hline 31 & $0,5,-$ & 7 & 6 & $9(\mathrm{M})$ & 22 & \\
\hline 32 & $0,0,0$ & 8 & 9 & & 22 & \\
\hline 33 & $0,0,-$ & 10 & 6 & & 23 & Y \\
\hline 34 & $0,0,0$ & 5 & 8 & 12 (Eq. 5(ii)) & 24 & \\
\hline 35 & $5,0,-$ & 5 & 11 & 15 (Eq. 14) & 25 & \\
\hline 36 & $0,5,0$ & 9 & 12 & $15\left(\mathrm{~A}_{35}\right)$ & 25 & \\
\hline 37 & $0,6,-$ & 11 & 15 & 16 (Eq. 5(ii)) & 31 & Y \\
\hline 38 & $0,0, \mathbf{1 9}$ & 13 & 12 & & 32 & Y \\
\hline 39 & $0,0,-$ & 17 & 18 & $19\left(\mathrm{~A}_{38}\right)$ & 33 & Y \\
\hline 40 & $0,0, \mathbf{2 0}$ & 14 & 18 & & 34 & $\mathrm{Y}$ \\
\hline 41 & $0,0,-$ & 12 & $24(\mathrm{R})$ & & 35 & Y \\
\hline 42 & $6,0,24$ & 12 & 22 & 27 (Eq. 5(ii)) & 36 & \\
\hline 43 & $0,13,-$ & 18 & 19 & 32 (Eq. 5(ii)) & 43 & \\
\hline 44 & $0,0,22$ & 19 & 27 & 38 (Eq. 5(ii)) & 44 & \\
\hline 45 & $0,0,-$ & 21 & 28 & $45(\mathrm{BE})$ & 45 & \\
\hline 46 & $0,0,23$ & 19 & 30 & $45\left(\mathrm{~A}_{45}\right)$ & 46 & \\
\hline 47 & $0,0,-$ & 23 & 31 & $45\left(\mathrm{~A}_{45}\right)$ & 47 & \\
\hline 48 & $48,0,48$ & 25 & $33(\mathrm{R})$ & & 48 & \\
\hline 49 & $49,56,-$ & 25 & $36(\mathrm{R})$ & $56(\mathrm{SS})$ & 56 & \\
\hline 50 & $50,49,50$ & 27 & 35 & $56\left(\mathrm{~A}_{49}\right)$ & 57 & \\
\hline 51 & $51,50,-$ & 28 & $38(\mathrm{R})$ & $56\left(\mathrm{~A}_{49}\right)$ & 58 & \\
\hline 52 & $52,51,52$ & 31 & $40(\mathrm{R})$ & $56\left(\mathrm{~A}_{49}\right)$ & 59 & \\
\hline 53 & $53,52,-$ & 33 & $40(\mathrm{R})$ & $56\left(\mathrm{~A}_{49}\right)$ & 60 & \\
\hline 54 & $54,53,54$ & 34 & 43 & $56\left(\mathrm{~A}_{49}\right)$ & 61 & \\
\hline 55 & $55,54,-$ & 32 & 44 & $57\left(\mathrm{H}_{49}\right)$ & 70 & \\
\hline 56 & $56,55,56$ & 32 & $47(\mathrm{R})$ & $57\left(\mathrm{~A}_{55}\right)$ & 72 & \\
\hline 57 & $57,56,-$ & 32 & $48(\mathrm{R})$ & & 73 & \\
\hline 58 & $\mathbf{5 8 , 5 7 , 5 8}$ & 34 & 47 & & 74 & Y \\
\hline 59 & $\mathbf{5 9}, 58,-$ & 32 & 51 & & 75 & Y \\
\hline 60 & $\mathbf{6 0}, 59, \mathbf{6 0}$ & 36 & 53 & & 77 & Y \\
\hline 61 & $\mathbf{6 1}, 70,-$ & 40 & $54(\mathrm{R})$ & & 87 & Y \\
\hline 62 & $62,61,62$ & 33 & 55 & 64 (Eq. 5(ii)) & 88 & Y \\
\hline 63 & $\mathbf{7 2}, 62,-$ & 33 & 57 & & 90 & $\mathrm{Y}$ \\
\hline
\end{tabular}

Table 10: Comparison of Results $d=12, w=7$. 


\begin{tabular}{|c|c|c|c|c|c|c|}
\hline$n$ & $C C, E C, Q C$ & $N B-M i x$ & $B-$ Lex & Misc & $U B$ & NewBest \\
\hline 29 & $0,14,-$ & 12 & 16 & 222 (Eq. 5(ii)) & 58 & \\
\hline 30 & $15,0,30$ & 12 & 17 & & 60 & Y \\
\hline 31 & $\mathbf{3 1}, 15,-$ & 12 & $21(\mathrm{R})$ & & 65 & $\mathrm{Y}$ \\
\hline 32 & $\mathbf{3 2}, 31, \mathbf{3 2}$ & 16 & 23 & & 88 & $\mathrm{Y}$ \\
\hline 33 & $\mathbf{3 3}, 32,-$ & 16 & $24(\mathrm{R})$ & & 90 & Y \\
\hline 34 & $\mathbf{3 4}, 33,{ }^{*} \mathbf{3 4}$ & 18 & $28(\mathrm{R})$ & & 97 & $\mathrm{Y}$ \\
\hline 35 & $35,34,-$ & 22 & 31 & & 105 & $\mathrm{Y}$ \\
\hline 36 & $36, \mathbf{4 0},{ }^{*} 36$ & 24 & 36 & & 112 & Y \\
\hline 37 & $37,36,-$ & 27 & $38(\mathrm{R})$ & $40\left(\mathrm{~A}_{36}\right)$ & 115 & Y \\
\hline 38 & $38,37,{ }^{*} 38$ & 26 & 39 & $40\left(\mathrm{~A}_{36}\right)$ & 147 & Y \\
\hline 39 & $39,38,-$ & 29 & $46(\mathrm{R})$ & 48 (Eq. 5(ii)) & 156 & Y \\
\hline 40 & $\mathbf{6 0}, 39, * 40$ & 33 & $50(\mathrm{R})$ & & 165 & Y \\
\hline 41 & & 35 & 53 & 64 (Eq. 5(ii)) & 174 & \\
\hline 42 & & 37 & 58 & 79 (Eq. 5(ii)) & 183 & \\
\hline 43 & & 40 & $64(\mathrm{R})$ & 96 (Eq. 5(ii)) & 193 & \\
\hline 44 & & 44 & 66 & 117 (Eq. 5(ii)) & 236 & \\
\hline 45 & & 47 & 72 & 142 (Eq. 5(ii)) & 247 & \\
\hline 46 & & 52 & 78 & 171 (Eq. 5(ii)) & 258 & \\
\hline 47 & & 56 & 85 & 205 (Eq. 5(ii)) & 270 & \\
\hline 48 & & 58 & $91(\mathrm{R})$ & 246 (Eq. 5(ii)) & 282 & \\
\hline 49 & & 61 & $99(\mathrm{R})$ & 294 (Eq. 5(ii)) & 294 & \\
\hline 50 & & 65 & 105 & 350 (SS) & 350 & \\
\hline 51 & & 71 & 114 & $350\left(\mathrm{~A}_{50}\right)$ & 363 & \\
\hline 52 & & 74 & $122(\mathrm{R})$ & $350\left(\mathrm{~A}_{50}\right)$ & 377 & \\
\hline 53 & & 81 & 131 & $350\left(\mathrm{~A}_{50}\right)$ & 390 & \\
\hline 54 & & 85 & $141(\mathrm{R})$ & $350\left(\mathrm{~A}_{50}\right)$ & 405 & \\
\hline 55 & & 91 & $152(\mathrm{R})$ & $350\left(\mathrm{~A}_{50}\right)$ & 419 & \\
\hline 56 & & 95 & $160(\mathrm{R})$ & $351\left(\mathrm{H}_{50}\right)$ & 490 & \\
\hline 57 & & 99 & 168 & $351\left(\mathrm{~A}_{56}\right)$ & 513 & \\
\hline 58 & & 106 & 178 & $351\left(\mathrm{~A}_{56}\right)$ & 529 & \\
\hline 59 & & 110 & 193 & $351\left(\mathrm{~A}_{56}\right)$ & 545 & \\
\hline 60 & & 118 & $204(\mathrm{R})$ & $352\left(\mathrm{H}_{56}\right)$ & 562 & \\
\hline 61 & & 123 & $216(\mathrm{R})$ & $352\left(\mathrm{~A}_{60}\right)$ & 587 & \\
\hline 62 & & 200 & 226 & $352\left(\mathrm{~A}_{60}\right)$ & 674 & \\
\hline 63 & & 224 & 244 & $352\left(\mathrm{~A}_{60}\right)$ & 693 & \\
\hline
\end{tabular}

Table 11: Comparison of Results $d=12, w=8$. 


\begin{tabular}{|c|c|c|c|c|c|c|}
\hline$n$ & $C C, E C, Q C$ & $N B-M i x$ & $B-L e x$ & Misc & $U B$ & NewBest \\
\hline 29 & $\overline{00,4,-}$ & $\overline{4}$ & 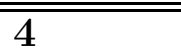 & & 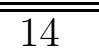 & \\
\hline 30 & $0,0,0$ & 4 & 4 & $5(\mathrm{M})$ & 15 & \\
\hline 31 & $0,0,-$ & 4 & 5 & & 15 & \\
\hline 32 & $4,0,4$ & 4 & 5 & & 16 & \\
\hline 33 & $0,4,-$ & 4 & 5 & $6(\mathrm{M})$ & 16 & \\
\hline 34 & $0,0,0$ & 4 & 5 & $6\left(\mathrm{~A}_{33}\right)$ & 17 & \\
\hline 35 & $0,0,-$ & 5 & 5 & $7(\mathrm{M})$ & 17 & \\
\hline 36 & $0,5,0$ & 5 & 6 & $9(\mathrm{M})$ & 22 & \\
\hline 37 & $0,0,-$ & 6 & 6 & $9\left(\mathrm{~A}_{36}\right)$ & 23 & \\
\hline 38 & $0,0,0$ & 6 & 6 & $9\left(\mathrm{~A}_{36}\right)$ & 23 & \\
\hline 39 & $0,0,-$ & 5 & 6 & $9\left(\mathrm{~A}_{36}\right)$ & 24 & \\
\hline 40 & $0,0,5$ & 5 & 6 & $10(\mathrm{NB})$ & 25 & \\
\hline 41 & $0,5,-$ & 5 & 7 & $10\left(\mathrm{~A}_{40}\right)$ & 25 & \\
\hline 42 & $0,0,0$ & 6 & 7 & $10\left(\mathrm{~A}_{40}\right)$ & 26 & \\
\hline 43 & $0,6,-$ & 8 & 10 & & 32 & \\
\hline 44 & $0,0,0$ & 9 & 12 & & 33 & $\mathrm{Y}$ \\
\hline 45 & $0,0,-$ & 12 & 7 & & 33 & $\mathrm{Y}$ \\
\hline 46 & $0,0,0$ & 13 & 9 & & 34 & $\mathrm{Y}$ \\
\hline 47 & $0,0,-$ & 6 & 12 & 15 (Eq. 5(ii)) & 35 & \\
\hline 48 & $6,0,6$ & 6 & $14(\mathrm{R})$ & 18 (Eq. 5(ii)) & 36 & \\
\hline 49 & $0,6,-$ & 11 & 15 & 21 (Eq. 5(ii)) & 36 & \\
\hline 50 & $0,7,0$ & 14 & 19 & 25 (Eq. 14) & 43 & \\
\hline 51 & $0,0,-$ & 15 & 14 & $25\left(\mathrm{~A}_{50}\right)$ & 44 & \\
\hline 52 & $0,0,26$ & 18 & 21 & 27 (Eq. 5(ii)) & 45 & \\
\hline 53 & $0,0,-$ & 19 & 22 & 31 (Eq. 5(ii)) & 46 & \\
\hline 54 & $0,0,27$ & 18 & 25 & 36 (Eq. 5(ii)) & 47 & \\
\hline 55 & $0,0,-$ & 15 & $27(\mathrm{R})$ & 42 (Eq. 5(ii)) & 48 & \\
\hline 56 & $7,0,28$ & 15 & 28 & 49 (Eq. 5(ii)) & 49 & \\
\hline 57 & $57,15,-$ & 24 & 23 & $57(\mathrm{SS})$ & 57 & \\
\hline 58 & $0, \mathbf{5 7}, 29$ & 23 & 33 & & 58 & \\
\hline 59 & $0,0,-$ & 25 & 33 & $57\left(\mathrm{~A}_{58}\right)$ & 59 & \\
\hline 60 & $0,0,30$ & 25 & $35(\mathrm{R})$ & $57\left(\mathrm{~A}_{58}\right)$ & 60 & \\
\hline 61 & $0,0,-$ & 27 & $37(\mathrm{R})$ & $57\left(\mathrm{~A}_{58}\right)$ & 61 & \\
\hline 62 & $0,0,31$ & 28 & $37(\mathrm{R})$ & $58\left(\mathrm{H}_{58}\right)$ & 62 & \\
\hline 63 & $63,0,-$ & 56 & $41(\mathrm{R})$ & & 63 & \\
\hline
\end{tabular}

Table 12: Comparison of Results $d=14, w=8$. 


\section{References}

[1] N.Q. A, L. Györfi and J.L. Massey. Constructions of binary constant-weight cyclic codes and cyclically permutable codes. IEEE Trans. Inform. Theory, vol. 38, (1992), 940-949.

[2] E. Agrell, A. Vardy, and K. Zeger. Upper bounds for constant-weight codes. IEEE Trans. on Inform. Theory, vol. 46, no. 7 (2000), 2373-2395.

[3] H.K. Aw, Y.M. Chee and A.C.H. Ling. Six new constant weight binary codes. Ars Combinatoria, vol. 67, (2003), 313-318.

[4] R.D. Baker. An elliptic semiplane. J. Combinatorial Theory A, vol. 25, (1978), 193195.

[5] G.T. Bogdanova and P.R.J. Östergård. Bounds on Codes over an Alphabet of Five Elements. Discrete Mathematics, vol. 240/1-3, (2001) 13-19.

[6] A.E. Brouwer, J.B. Shearer, N.J.A. Sloane and W.D. Smith. A new table of constant weight codes. IEEE Trans. Inform. Theory, vol. 36, no. 6, (1990), 1334-1380.

[7] R. Carraghan and P.M. Pardalos. An exact algorithm for the maximum clique problem. Operations Research Letters, 9(1990) 375-382.

[8] C.J. Colbourn and J.H. Dinitz. The CRC handbook of combinatorial designs. Boca Raton, Florida, CRC Press, 1996.

[9] J.H. Conway and N.J.A. Sloane. Lexicographic codes; error-correcting codes from game theory. IEEE Trans. Inform. Theory, vol. 32,(1986), 337-348.

[10] F.J. MacWilliams and N.J.A. Sloane. The theory of error-correcting codes. Amsterdam, The Netherlands, North-Holland, 1977.

[11] J.N.J. Moon, L.A. Hughes and D.H. Smith. Assignment of frequency lists in frequency hopping networks. IEEE Trans. on Vehicular Technology, vol. 54, no. 3, (2005), 11471159.

[12] D.H. Smith, A. Sakhnovich, S. Perkins, D.G. Knight and L.A. Hughes. Application of coding theory to the design of frequency hopping lists. Technical Report UG-M-02-1, University of Glamorgan, 2002. Available at http://www.glam.ac.uk/sot/doms/Research/radiofreq.php

[13] Table of constant weight binary codes.

http://www.research.att.com/ ${ }^{\sim}$ njas/codes/Andw

[14] Table of bounds for constant weight binary codes. http://www.s2.chalmers.se/ ${ }^{\sim}$ agrell/bounds/cw.html

[15] http://www.mathi.uni-heidelberg.de/ ${ }^{\sim}$ yves/Matritzen/CWCodes/CWCode.html 